\title{
A Multivariate Regression Approach to Adjust AATSR Sea Surface Temperature to In Situ Measurements
}

\author{
Pierre Tandeo, Emmanuelle Autret, Jean François Piollé, Jean Tournadre, and Pierre Ailliot
}

\begin{abstract}
The Advanced Along-Track Scanning Radiometer (AATSR) onboard Envisat is designed to provide very accurate measurements of sea surface temperature (SST). Using colocated in situ drifting buoys, a dynamical matchup database (MDB) is used to assess the AATSR-derived SST products more precisely. SST biases are then computed. Currently, Medspiration AATSR SST biases are discrete values and can introduce artificial discontinuities in AATSR level-2 SST fields. The new AATSR SST biases presented in this letter are continuous. They are computed, for nighttime and best proximity confidence data, by linear regression with different MDB covariables (wind speed, latitude, aerosol optical depth, etc.). As found, the difference between dual-view and nadir-only SST products explains most of the variability $(26 \%)$.
\end{abstract}

Index Terms-Advanced Along-Track Scanning Radiometer (AATSR), remote sensing, sea surface temperature (SST), validation.

\section{INTRODUCTION}

$\mathbf{T}$ HE ALONG-TRACK Scanning Radiometer (ATSR) series [including the Advanced ATSR (AATSR)] is a generation of infrared radiometers which were built specifically to retrieve accurate measurements of sea surface temperature (SST) for environmental and climate applications. The AATSR onboard Envisat is a fundamental data set used within the Godae High Resolution Sea Surface Temperature Pilot Project (GHRSST-PP) analysis systems (see [1]) as the reference data set to estimate and correct bias with other satellite data prior to merging and objective analysis processes.

An optimal assignment of error and confidence for each measurement is indeed essential to merging and objective analysis. In the case of infrared SST data sets, data are categorized according to wind speed (used to identify favorable conditions to sea surface stratification) and their proximity to clouds. Bias and standard deviation errors [also called sensor-specific error statistics (SSES)] estimated by statistical analysis of in situ satellite matchups are then assigned to the SST samples.

Manuscript received August 5, 2008; revised September 10, 2008. First published November 11, 2008; current version published January 14, 2009. This work was supported by the Medspiration Project by the European Space Agency.

P. Tandeo, E. Autret, J. F. Piollé, and J. Tournadre are with the Laboratoire d'Océanographie Spatiale, Institut Français de Recherche pour l'Exploitation de la Mer (IFREMER), 29280 Plouzané, France (e-mail: pierre.tandeo@ifremer.fr; emmanuelle.autret@ifremer.fr; jfpiolle@ifremer.fr; jean.tournadre@ifremer.fr).

P. Ailliot is with the Département de Mathématiques, Université de Bretagne Occidentale, 29200 Brest, France (e-mail: pierre.ailliot@univ-brest.fr).

Digital Object Identifier 10.1109/LGRS.2008.2006568
A proximity confidence value is then eventually assigned based on its "proximity" to several criteria known to have effects on the final SST estimate.

The current AATSR SSES (see [2]) given by Medspiration (the European node to GHRSST system; http://www. medspiration.org) are stratified in a 12-fold clustering of data, corresponding to different values of covariates. Among them, moderate to high wind speed $\left(>6 \mathrm{~m} \cdot \mathrm{s}^{-1}\right)$ is identified to have an impact on the mixed layer and to reduce SST variability. Moreover, the difference between the two possible AATSR SST measurements, i.e., the dual-view (combination of nadir and $55^{\circ}$ incidence view; see [3] for more details) and nadir-only SST retrievals, is split into three categories. The dual-nadir SST difference allows the detection of possible contamination by clouds, aerosols, or other contaminants. Finally, the well-known problem of diurnal cycle is solved by splitting the data into nighttime and daytime measurements. The corresponding 12 biases and standard deviations of the SSES have been computed from the colocated data stored in the matchup database (MDB) presented in Section II. Applying such discrete biases can introduce artificial discontinuities in AATSR level-2 (L2) SST fields which can cause instabilities in the interpolated analysis.

Our objective is to define and determine continuous AATSR SSES based on the same MDB as the segmented Medspiration AATSR biases. We focus on the SST measurements currently used by most of the AATSR SST users, i.e., nighttime and best proximity confidence data. The regression method used to compute continuous bias values is described in Section III. Section IV presents the results of the MDB analysis in terms of SSES, as well as a comparison between the derived AATSR SST biases. Section V gives some concluding remarks and perspectives for future work.

\section{DATA}

The development of the GHRSST-PP system has strengthened the need for the validation and intercomparison of satellite SST products. In situ data are a reliable independent source of measurements widely used to validate these products (see [4]), and the Institut Français de Recherche pour l'Exploitation de la Mer (IFREMER), Brest, France, operationally produces an MDB (http://www.medspiration.org/tools/mdb/) that provides colocated satellite and in situ measurements. The system relies on the Coriolis system (for the worldwide collection and 


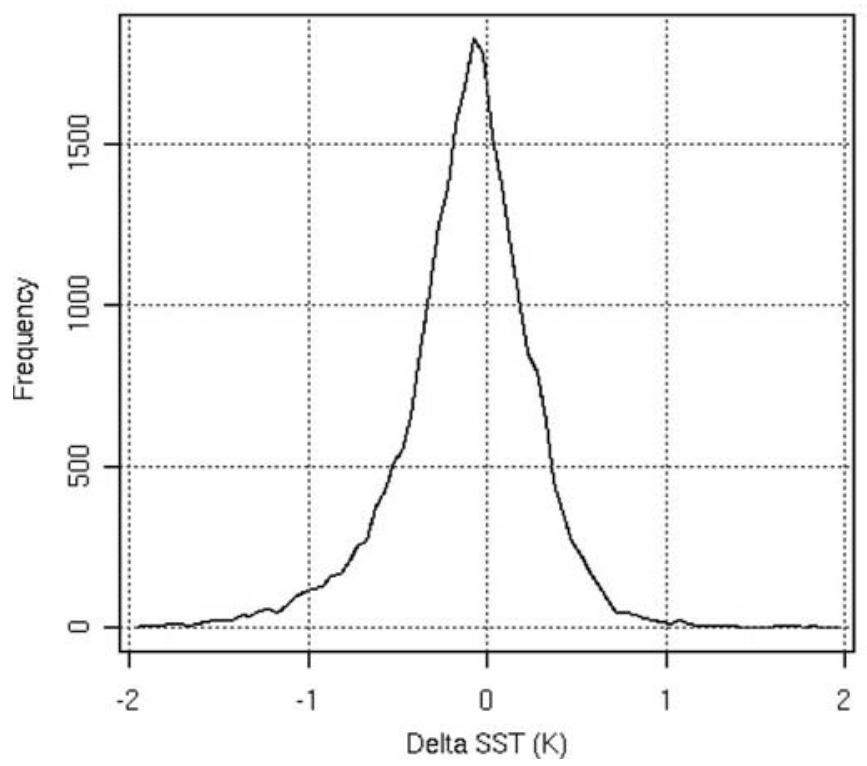

Fig. 1. Distribution of the AATSR $\Delta$ SST for nighttime best quality pixels. The mean bias is $-0.11 \mathrm{~K}$ with an std of $0.39 \mathrm{~K}$.

archiving of in situ sea data; http://www.coriolis.eu.org) for in situ measurements and on the Medspiration archive for satellite data, both hosted at IFREMER.

Assuming that the drifting-buoy measurements constitute the sea truth, the accuracy of AATSR SST, $\Delta$ SST, is defined as

$$
\Delta \mathrm{SST}=\mathrm{SST}_{\text {dual }}-\mathrm{SST}_{\text {buoys }}
$$

where $\mathrm{SST}_{\text {dual }}$ is the dual-view skin SST estimation given in the AATSR L2 products and SST buoys is the temperature measured by drifting buoys at a depth that depends on the buoy type (is generally close to $1 \mathrm{~m}$ ) that is called subskin SST. They are provided by the Coriolis system where they are quality controlled and archived. The skin-to-subskin adjustment presented by [5] is not used here as it will be directly integrated into our model.

The best quality pixels, i.e., nighttime colocated data with the highest proximity confidence value (i.e., $\mathrm{SST}_{\text {dual }}-\mathrm{SST}_{\text {nadir }} \in$ $\{-0.51,0.51\} \mathrm{K})$, are taken into account. Colocation criteria are $\Delta x<10 \mathrm{~km}, \Delta t<2 \mathrm{~h}$, and $|\Delta \mathrm{SST}|<2 \mathrm{~K}$. The latter criterion is used to eliminate obvious outliers that can strongly modify the standard deviation. The data set contains 25384 matchups. The corresponding distribution of $\Delta$ SST is shown in Fig. 1.

In addition to $\Delta \mathrm{SST}$, the MDB also provides covariates from different sources such as model outputs or ancillary fields about data quality. Nine covariates are available, and their descriptions are given in Table I. Among them, the two covariates providing statistics on the satellite pixels surrounding the in situ measurement are computed within a $25 \times 25 \mathrm{~km}^{2}$ box. They give a contextual information on the satellite data quality and variability and/or possible cloud contamination.

The MDB used in this letter covers two and a half years (from April 2005 to September 2007) and most of the global ocean. It should, however, be noted that the Atlantic Ocean is
TABLE I

DESCRIPTION OF THE MDB COVARIATES USED IN THIS LETTER

\begin{tabular}{ccc}
\hline Name & Description & Source \\
\hline Longitude & Measurement longitude & L2 \\
Latitude & Measurement latitude & L2 \\
Aerosol optical depth & Aerosol atmosphere quantity & NAAPS \\
Wind speed & Near surface wind speed & ECMWF \\
Zenithal solar angle & Angle between zenith and sun & L2 \\
Valid pixel in box & Valid pixel by $25 \times 25 \mathrm{~km}^{2}$ box & Ifremer \\
Sigma SST in box & Std SST by $25 \times 25 \mathrm{~km}^{2}$ box & Ifremer \\
$S S T_{\text {dual }}$ & SST dual view & L2 \\
$S S T_{\text {dual }}-S S T_{\text {nadir }}$ & Dual-nadir SST difference & L2
\end{tabular}

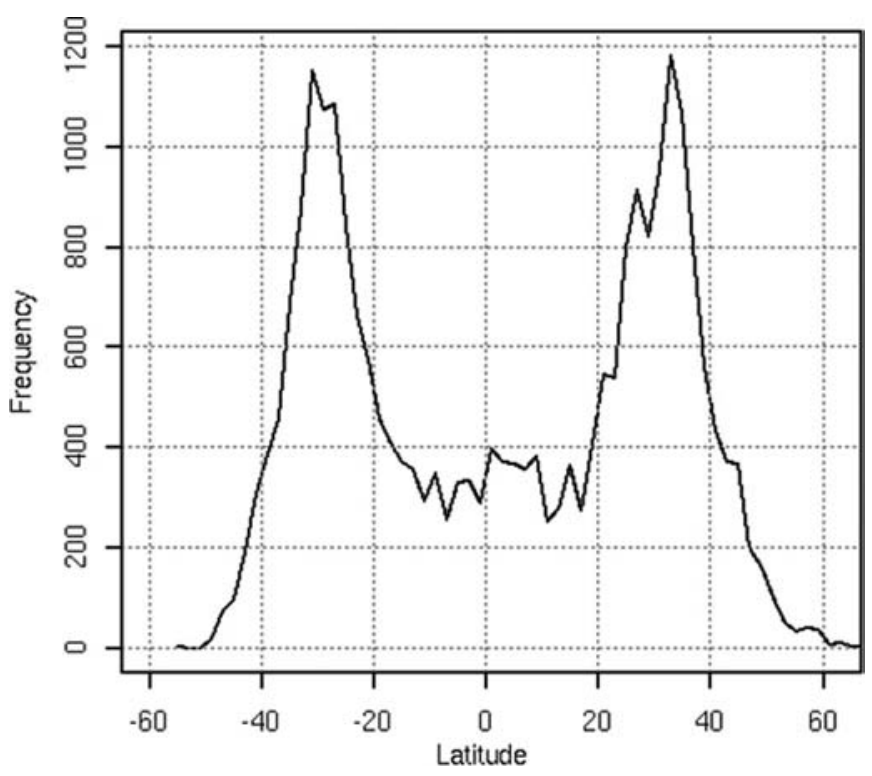

Fig. 2. Number of colocated samples by band of $2^{\circ}$ latitude.

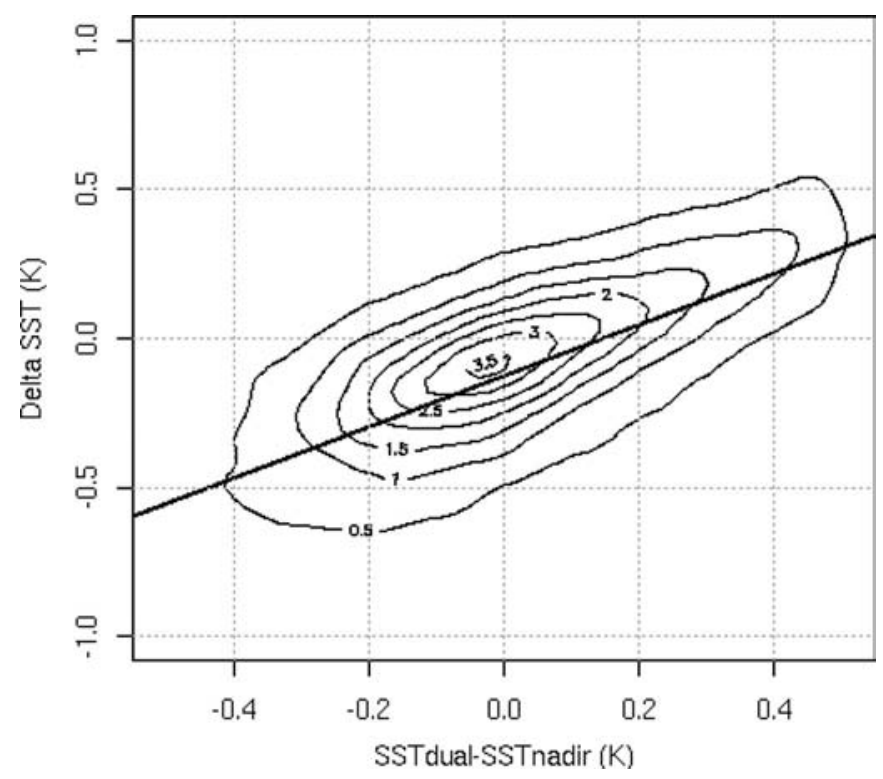

Fig. 3. Bidimensional pdf of $\Delta$ SST and AATSR dual-nadir SST difference and the corresponding regression line (intercept $=-0.13$, slope $=0.86$, and $R^{2}=26 \%$ )

overrepresented because of a higher availability of in situ data. Moreover, Fig. 2 shows that the sampling in latitude is irregular with a relatively small number of colocated data in the tropical 
TABLE II

Coefficients of the Model for the Selected Covariate (Nighttime Measurements OnLy)

\begin{tabular}{ccc}
\hline Covariate & Associated parameter value & Confidence interval (95\%) \\
\hline Intercept $\alpha^{(0)}$ & 0.006 & {$[-0.005 ; 0.016]$} \\
Sigma SST in box $\alpha^{(1)}$ & -0.422 & {$[-0.452 ;-0.392]$} \\
Zenithal solar angle $* S S T_{\text {dual }}-S S T_{\text {nadir }} \beta^{(1)}$ & 0.007 & {$[0.0068 ; 0.0071]$}
\end{tabular}

TABLE III

Coefficients of the Model for the Selected Covariate (Daytime Measurements Only)

\begin{tabular}{ccc}
\hline Covariate & Associated parameter value & Confidence interval (95 \%) \\
\hline Intercept $\alpha^{\prime(0)}$ & 0.039 & {$[0.018 ; 0.061]$} \\
Sigma SST in box * SST $T_{\text {dual }} \beta^{\prime(1)}$ & 0.030 & {$[0.027 ; 0.032]$} \\
Sigma SST in box $* S S T_{\text {dual }}-S S T_{\text {nadir }} \beta^{\prime(2)}$ & 1.112 & {$[0.972 ; 1.252]$}
\end{tabular}

band and a large number at midlatitude. The model that can be estimated from the MDB cannot be mainly representative of high-latitude conditions $\left(>40^{\circ}\right)$.

\section{METHOD}

Our aim is to estimate $\Delta$ SST in real time in order to adjust $\mathrm{SST}_{\text {dual }}$ measurements. In statistical terms, it is equivalent to finding the effects of simultaneous $p$ covariates $\left\{X^{(i)}, i=\right.$ $1, \ldots, p\}$ on a response variable $\Delta$ SST. The analysis of the bidimensional probability density function (pdf) of $\Delta \mathrm{SST}$ and $\mathrm{SST}_{\text {dual }}-\mathrm{SST}_{\text {nadir }}$, shown in Fig. 3, shows that the relationship between the two variables is quite linear. The pdfs of $\triangle$ SST and the other covariates (not shown here) present very similar features. Therefore, a simple linear model, which will thus implicitly include a skin-to-subskin adjustment, is used to model $\Delta S S T$ as a function of the covariates. The simplest linear model can be expressed as

$$
\Delta \mathrm{SST}=\alpha^{(0)}+\sum_{i=1}^{p} \alpha^{(i)} X^{(i)}+\varepsilon
$$

where $\varepsilon$ is a Gaussian white noise and $X^{(i)}$ denotes the $p$ covariates. The coefficients $\alpha^{(0)}$ and $\alpha^{(i)}$ are the intercept and principal effects on covariates, respectively. However, some covariate effects might be linked, and their possible interaction can be taken into account by including quadratic terms in the model in the form

$\Delta \mathrm{SST}=\alpha^{(0)}+\sum_{i=1}^{p} \alpha^{(i)} X^{(i)}+\sum_{i=1}^{p} \sum_{j=1}^{p} \beta^{(i: j)} X^{(i)} X^{(j)}+\varepsilon$

where $\beta^{(i: j)}$ corresponds to the interaction between the covariates $X^{(i)}$ and $X^{(j)}$. All the parameters in (3) are estimated by the least squares method. In order to select the best fit model with fewer parameters, we conducted an exhaustive search based on the best fit criterion $R_{\text {adj }}^{2}$ defined as

$$
R_{\text {adj }}^{2}=1-\frac{\operatorname{Var}_{\text {error }}}{\operatorname{Var}_{\text {total }}}
$$

where

$$
\begin{aligned}
\operatorname{Var}_{\text {error }} & =\frac{\sum(\Delta \mathrm{SST}-\Delta \widehat{\mathrm{SST}})^{2}}{n-p-1} \\
\operatorname{Var}_{\text {total }} & =\frac{\sum(\Delta \mathrm{SST}-\Delta \overline{\mathrm{SST}})^{2}}{n-1}
\end{aligned}
$$

with $\Delta \widehat{\mathrm{SST}}$ being the $\Delta \mathrm{SST}$ estimated by the model, $\Delta \overline{\mathrm{SST}}$ being the mean of $\Delta$ SST, and $n$ being the number of observations. $R_{\text {adj }}^{2}$ gives an information about the quality of the fit with a penalization on $p$ (the complexity), the number of parameters estimated in the model.

\section{RESUlTS}

An exhaustive search of the model with all covariate combinations is done in accordance with the $R_{\text {adj }}^{2}$ introduced earlier. Model selection shows that the interaction between the zenithal solar angle and $\mathrm{SST}_{\text {dual }}-\mathrm{SST}_{\text {nadir }}$ is the most important effect on $\triangle$ SST. The addition of the standard deviation of SST by validation box of $25 \times 25 \mathrm{~km}^{2}$ around the pixel improves the adjustment $\left(R_{\mathrm{adj}}^{2}=28 \%\right)$. Owing to the small increase of $R_{\mathrm{adj}}^{2}$ in adding new covariates ( $\mathrm{SST}_{\text {dual }}$, aerosol optical depth, etc.), the simple model with three parameters (including intercept) is chosen. The $\alpha$ and $\beta$ parameters and their confidence interval corresponding to those variables are given in Table II. This model identifies the dual-nadir difference retrieval as the most important covariate. It explains $26 \%$ of the residual SST variability (see Fig. 3).

Although daytime data are rarely used for objective analysis purposes (mainly because the correction of diurnal warming is not fully solved), the method has also been applied to best quality flag daytime data. The data set contains 7931 matchups. Three parameters are also retained and are given in Table III. The residual SST variability $\left(R_{\text {adj }}^{2}=\right.$ $15.3 \%)$ is less explained certainly because, even for the best quality sample, diurnal effect cannot be completely eliminated.

The SSES model has been tested on real-time data, i.e., AATSR L2 files corresponding to one day (May 24, 2008) with nighttime measurements and best quality pixel in North Atlantic, corresponding to a small difference between dualview and nadir-only SST retrievals $\left(\left|\mathrm{SST}_{\text {dual }}-\mathrm{SST}_{\text {nadir }}\right|<\right.$ $0.51 \mathrm{~K})$ and, therefore, small cloud or aerosol contaminants 


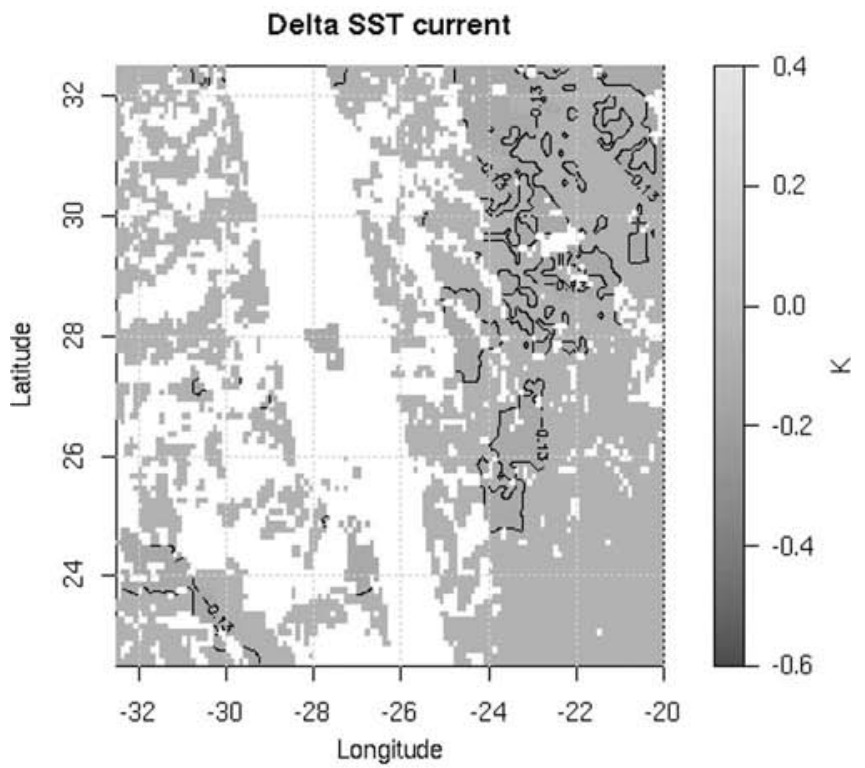

(a)

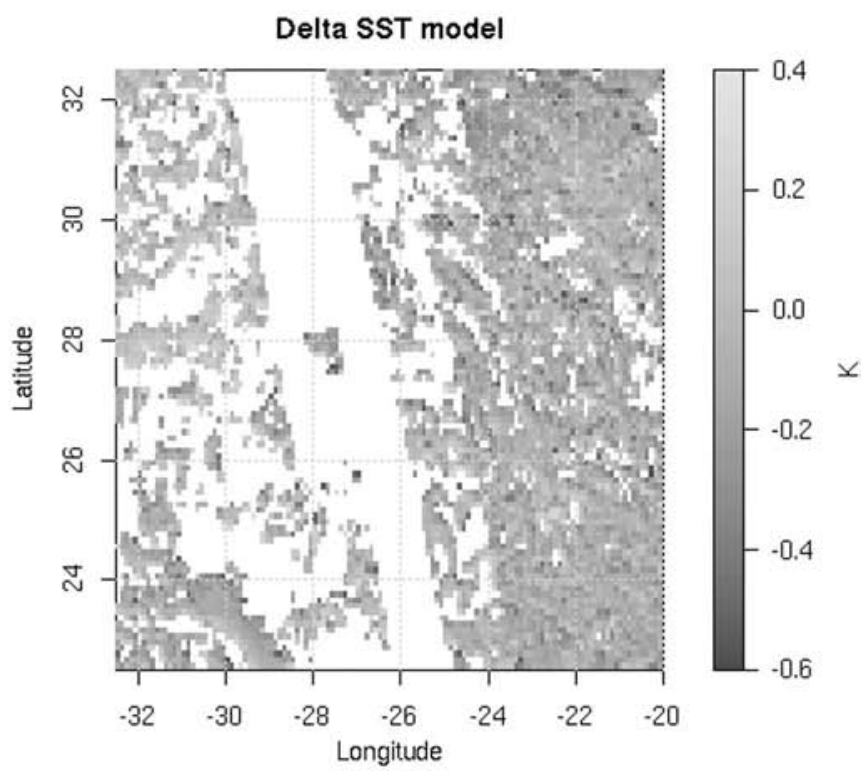

(b)

Fig. 4. (a) $\Delta \widehat{S S T}_{\text {current }}$ given in the AATSR L2 files in a part of North Atlantic for nighttime measurements and best quality pixels (May 26, 2008; contour plot identifies the two values, namely, $-0.13 \mathrm{~K}$ and $-0.07 \mathrm{~K}$ ). (b) $\Delta \widehat{\mathrm{SST}}_{\text {model }}$ computed by model (3) on the same data.

of the $\mathrm{SST}_{\text {dual }}$. Fig. 4 compares the original $\Delta \widehat{\mathrm{SST}}_{\text {current }}$ and $\Delta \widehat{\mathrm{SST}}_{\text {model }}$ computed by the model proposed in Table II. For these particular data, $\Delta \widehat{\mathrm{SST}}_{\text {current }}$ takes only two values, namely, -0.13 and -0.07 , corresponding to low- and highwind-speed $\left(>6 \mathrm{~m} \cdot \mathrm{s}^{-1}\right)$ conditions, respectively. Both values include the $-0.17-\mathrm{K}$-depth-to-skin adjustment proposed by [5]. The figure also shows that discrete biases can create artificial SST gradients, while the new model gives continuous $\Delta \widehat{\mathrm{SST}}_{\text {model }}$, as shown in Fig. 4(b).

The current and model SST biases are also validated by comparison to an independent data set constituted by the SST measured by the infrared Advanced Very High Resolution Radiometer (AVHRR) sensor onboard METOP-A. The adjusted SSTs are defined by

$$
\mathrm{SST}_{\mathrm{adj}}=\mathrm{SST}_{\mathrm{dual}}-\Delta \widehat{\mathrm{SST}}
$$

where $\Delta \mathrm{SST}$ is the current or model bias. Only nighttime, best confidence value, and $\Delta t<3 \mathrm{~h}$ colocated data are studied. We test the model on three days in the year under different conditions representing 197688 pixels. Fig. 5 shows the pdfs of the difference between AVHRR SST and the nonadjusted (without any bias) and adjusted (current and model) AATSR dual SST. The distribution of the AVHRR and dual SST AATSR is, as expected, positively biased $(0.09 \mathrm{~K})$ because no subskinto-skin correction is applied. The adjustment of AATSR SST by the current SSES strongly reduces the mean bias to $-0.004 \mathrm{~K}$, but the distribution has the same standard deviation $(0.42 \mathrm{~K})$. The model adjustment further reduces the standard deviation (0.36 K).

\section{Conclusion And Perspective}

The MDB maintained at IFREMER within the Medspiration project to colocate satellite and in situ SST measurements

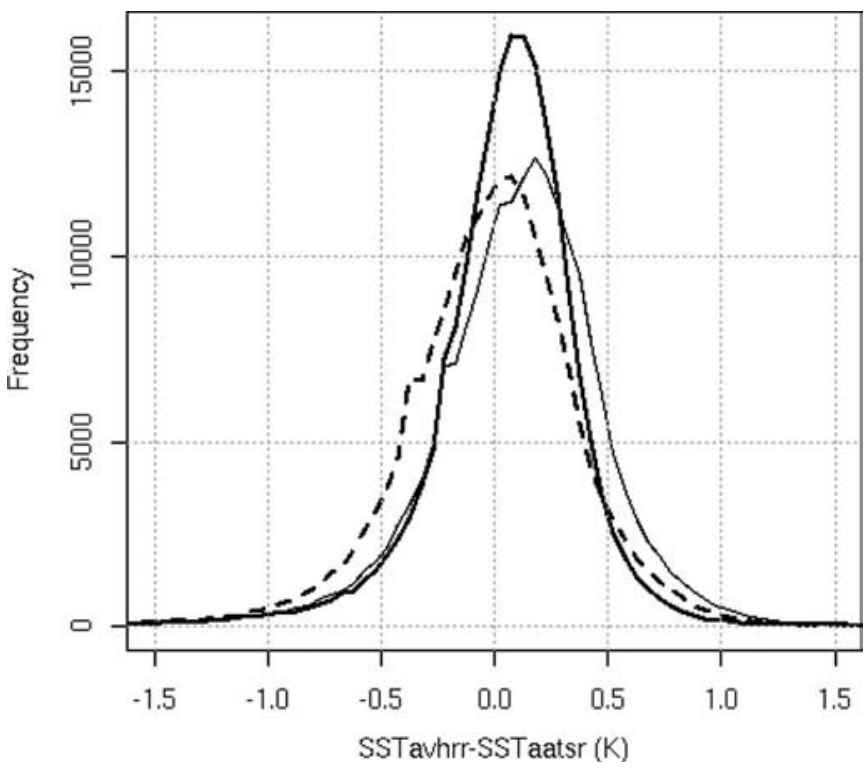

Fig. 5. Histograms of the difference between the AVHRR SST and (thick solid line) $\widehat{\mathrm{SST}}_{\text {model }}$, (dashed line) $\widehat{\mathrm{SST}}_{\text {current }}$, and (thin solid points) $\mathrm{SST}_{\text {dual }}$ for the 197688 colocated pixels in the Atlantic Ocean on several days (January 20, May 26, and August 17, 2008). Only nighttime best quality pixels are considered.

has been used to estimate a continuous model of SST bias $\left(\Delta \mathrm{SST}_{\text {model }}\right)$ for the AATSR sensor. $\Delta \mathrm{SST}_{\text {model }}$ has been fitted by linear regression on nine covariates. Among them, the $\mathrm{SST}_{\text {dual }}-\mathrm{SST}_{\text {nadir }}$ explains a large part of the $\Delta \mathrm{SST}$ variability. The continuous nature of the model biases means that it is well adapted for merging and objective analysis processes while being similar to the categorized $\Delta \mathrm{SST}_{\text {current }}$ given by Medspiration.

Nevertheless, the use of the MDB in order to fit a model on SST bias is questionable. First, we use drifting-buoy 
measurements as reference which measure the subskin SST. Second, we point the lack of sampling in high latitude. An upcoming study will evaluate the latitude distribution of AATSR L2 files in order to reproduce a similar distribution in a new MDB. This artificial variability in low sampled latitudes can be created by bootstrap methods. Finally, we need a more elaborate study on the colocation parameters (distance and time difference) between the AATSR sensor and drifting buoys in order to specify the data sampling.

As derived, the method presented in this letter can also be applied to instruments such as the AVHRR and the Advanced Microwave Scanning Radiometer which are also available in the MDB.

\section{ACKNOWLEDGMENT}

The authors would like to thank Dr. G. Corlett for his help during this work.

\section{REFERENCES}

[1] The GHRSST-PP Science Team and ISDI-TAG Working Group, The Recommended GHRSST-PP Data Processing Specification GDS. No. GHRSST/17, Mar. 2004. [Online]. Available: http://ghrsst-pp. metoffice.com

[2] G. K. Corlett and D. J. Poulter, "An updated confidence flagging scheme for Medspiration AATSR L2P files," Univ. Leicester, U.K., Tech. Rep., MED-UL-REP-002-1D, Medspiration, 2008. [Online]. Available: http://medspiration.org

[3] D. Llewellyn-Jones, M. C. Edwards, C. T. Mutlow, A. R. Birks, I. J. Barton, and H. Tait, "AATSR: Global-change and surface-temperature measurements from Envisat," ESA Bull., vol. 105, pp. 11-21, Feb. 2001.

[4] G. K. Corlett, I. J. Barton, C. J. Donlon, M. C. Edwards, S. A. Good, L. A. Horrocks, D. T. Llewellyn-Jones, C. J. Merchant, P. J. Minnett, T. J. Nightingale, E. J. Noyes, A. G. O'Carroll, J. J. Remedios, I. S. Robinson, R. W. Saunders, and J. G. Watts, "The accuracy of SST retrievals from AATSR: An initial assessment through geophysical validation against in situ radiometers, buoys and other SST data sets," Adv. Space Res., vol. 37, no. 4, pp. 764-769, 2006.

[5] C. J. Donlon, P. J. Minnett, C. Gentemann, T. J. Nightingale, I. J. Barton, B. Ward, and M. J. Murray, "Toward improved validation of satellite sea surface skin temperature measurements for climate research," J. Clim., vol. 15, no. 4, pp. 353-369, Feb. 2002. 